\title{
EXPLOITING INDEPENDENCE FOR CO-CHANNEL INTERFERENCE CANCELLATION AND SYMBOL DETECTION IN MULTIUSER DIGITAL COMUNICATIONS
}

\author{
Vicente Zarzoso \\ Signal Processing and Communications Group, Department of Electrical Engineering and Electronics \\ The University of Liverpool, Brownlow Hill, Liverpool L69 3GJ, UK \\ Tel/Fax: +44 (0)151 79445 32/40; e-mail: vicente@liverpool.ac.uk
}

\begin{abstract}
In the blind equalization of multi-input multi-output (MIMO) finite impulse response communication channels, co-channel interference (CCI) is typically cancelled by exploiting the properties of digital modulations, such as their finite alphabet (FA). This contribution takes advantage of the mutual independence of the users' signals through the application of independent component analysis (ICA). We demonstrate that ICA-based CCI suppression remarkably improves an FA-based approach. In addition, proposed ICA-assisted minimum mean square error receivers are shown to enhance the conventional detection capabilities of MIMO equalization methods relying on channel identification. The particular structure of the MIMO model yields a simplified detection scheme with improved performance at a reduced computational cost.
\end{abstract}

\section{INTRODUCTION}

Owing to their fundamental relevance in current as well as future wireless networks, communication systems where multiple users simultaneously transmit digital information through a common transmission medium have become a major focus of research attention. In such scenarios, co-channel interference (CCI) caused by other users adds to the intersymbol interference (ISI) generated by multipath propagation, hindering the reception of the signal(s) of interest. Space-time equalization techniques are required to mitigate the channel effects, in terms of both ISI and CCI, thus improving signal detection at the receiving end. Although training sequences can be used, proceeding blindly proves clearly advantageous [1], which gives rise to the blind channel identification and equalization (BIE) problem.

When spatio-temporal diversity is exploited in the form of multiple antennas and/or time oversampling, the sensor output adopts the so-called multi-input multi-output (MIMO) signal model. Typically, the multipath propagation effects are well approximated by finite impulse response (FIR) filters, and then the MIMO model is characterized by a channel matrix composed of the polyphase coefficients. Hence, one can first identify the channel matrix and then employ a conventional detector, such as zero-forcing or minimum mean square error (MMSE), to obtain the source symbols from the channel estimate [2, 3, 4]. The main drawback of this approach is the requirement of estimating a 'nuisance' parameter such as the channel matrix, when the actual parameters of interest are, in most cases, the users' symbols. As a result, inaccuracies in the channel estimate have a detrimental effect in the detection performance. Direct symbol detection without channel estimation

The author is supported through a Post-Doctoral Research Fellowship awarded by the Royal Academy of Engineering of the UK. is also feasible [1, 5]. This type of methods treat the source data as deterministic sequences, and so the benefit of sparing the channel estimation is attained at the expense of a loss of consistency with respect to the sample size [5].

A two-stage processing is necessary for space-time equalization [1, 5, 6]. In the first stage, second-order statistics (SOS) are able to cancel ISI by taking advantage of the structural properties of the channel and the source data (or correlation) matrices. An instantaneous linear mixture of the source signals (i.e., a CCI-only cancellation problem) is resolved in a second stage. To achieve the source separation, most proposed methods exploit properties inherent to digital signals, such as their constant modulus (CM) or finite alphabet (FA). For instance, the family of iterative least squares algorithms - with projection (ILSP), with enumeration (ILSE), and with subspace fitting (ILSF) [1, 5, 7] - utilize the FA property. For CM signals [8] or simple modulations (BPSK) [9] closed-form solutions are available, but global convergence of the ILSx-type of methods is not generally guaranteed [1, 7], even in the absence of noise [10]. In techniques relying on previous channel identification, the above ISI/CCI-removal steps are implicitly carried out when estimating the channel matrix, and actually take effect at detection.

The present contribution exploits another very plausible property: the statistical independence of the users' signals. We demonstrate by computer simulations that benefiting from source independence through the use of an independent component analysis (ICA) tool can lead to dramatic improvements in CCI-cancellation performance, compared to an FA-based method. In addition, it was recently proved [11] that an ICA-based refinement can enhance the robustness of conventional data detection in a particular directsequence code-division multiple access (DS-CDMA) model. We verify in this paper that analogous conclusions apply in the more generic FIR-MIMO framework, where ICA-assisted MMSE detection schemes are shown to compensate for channel estimation errors, yielding remarkable performance gains relative to the conventional MMSE receiver.

Notations. Symbol $\mathbf{I}_{n}$ refers to the $n \times n$ identity matrix; $(\cdot)^{\mathrm{T}},(\cdot)^{\mathrm{H}}$ and $(\cdot)^{-1}$ indicate the transpose, Hermitian (conjugatetranspose) and inverse matrix operators, respectively; $\mathrm{E}[\cdot]$ represents the mathematical expectation; $\otimes$ and $\odot$ stand for the Kronecker and elementwise product, respectively.

\section{SIGNAL MODEL}

Let us consider a digital communications system where: (A1) $K$ users transmit, at a known constant rate $1 / T_{s}$, zero-mean unit-variance mutually-independent information-bearing i.i.d. symbols $\mathbf{s}(n)=\left[s_{1, n}, \ldots, s_{K, n}\right]^{\mathrm{T}} \in \mathbb{C}^{K}$; 
(A2) the receiving sensor, with a spatio-temporal diversity (product of antenna elements and oversampling factor) of $L$, outputs vector samples $\mathbf{x}(n)=\left[x_{1, n}, \ldots, x_{L, n}\right]^{\mathrm{T}} \in \mathbb{C}^{L}$;

(A3) the channel effects (including transmission and propagation delays, phase shifts, amplitude attenuation, CCI and multipathinduced ISI) can be modelled by means of $M$ th-order FIR filters with matrix coefficients $\mathbf{H}(k) \in \mathbb{C}^{L \times K}, k=0,1, \ldots, M$;

(A4) the zero-mean additive noise $\mathbf{v}(n) \in \mathbb{C}^{L}$ at the sensor output has covariance matrix $\sigma^{2} \mathbf{I}_{L}$ and is independent of the transmitted symbols.

Under the above assumptions, the MIMO model can be expressed as:

$$
\mathbf{x}(n)=\sum_{k=0}^{M} \mathbf{H}(k) \mathbf{s}(n-k)+\mathbf{v}(n)
$$

with $n=M, \ldots, N_{d}+M-1$, where $N_{d}$ represents the number of observed symbol periods. Stacking $N$ consecutive received signal vector samples leads to the matrix model:

$$
\mathbf{x}_{n}=\mathbf{H} \mathbf{s}_{n}+\mathbf{v}_{n}
$$

with $\mathbf{s}_{n}=\left[\mathbf{s}(n+N-1)^{\mathrm{T}}, \mathbf{s}(n+N-2)^{\mathrm{T}}, \ldots, \mathbf{s}(n-M)^{\mathrm{T}}\right]^{\mathrm{T}}$,

$$
\mathbf{H}=\left[\begin{array}{cccccc}
\mathbf{H}(0) & \ldots & \mathbf{H}(M) & 0 & \cdots & 0 \\
0 & \mathbf{H}(0) & \cdots & \mathbf{H}(M) & \cdots & 0 \\
\vdots & \ddots & \ddots & \ddots & \ddots & \vdots \\
0 & \ldots & 0 & \mathbf{H}(0) & \cdots & \mathbf{H}(M)
\end{array}\right]
$$

$\mathbf{x}_{n}=\left[\mathbf{x}(n+N-1)^{\mathrm{T}}, \mathbf{x}(n+N-2)^{\mathrm{T}}, \ldots, \mathbf{x}(n)^{\mathrm{T}}\right]^{\mathrm{T}}$, and a similar definition for $\mathbf{v}_{n}, n=M, \ldots, N_{d}+M-N$. We also assume in the sequel that the block-Toeplitz channel matrix $\mathbf{H} \in \mathbb{C}^{L N \times K(M+N)}$ is full column rank, which imposes obvious necessary restrictions on its dimensions. More elaborate sufficient conditions are given in [6].

The objective of blind channel identification is to estimate the channel matrix $\mathbf{H}$ from the only observation of sensor vectors $\left[\mathbf{x}(M), \ldots, \mathbf{x}\left(N_{d}+M-1\right)\right]$. Blind equalization is concerned with the estimation of the transmitted data symbols $\mathbf{S}=$ $\left[\mathbf{s}(0), \ldots, \mathbf{s}\left(N_{d}+M-1\right)\right]$ from the receiving sensor output.

\section{ICA-BASED CCI CANCELLATION}

The extension to the multiuser case of BIE methods originally designed for the single-user case resolves ISI but is unable to cancel CCI. The blind channel identification method of [2] is based on the special structure exhibited by the source autocorrelation matrices in the i.i.d. case. It is proved in [4] that in the multiuser case the estimated channel matrix is of the form $\hat{\mathbf{H}}=\mathbf{H}\left(\mathbf{I}_{M+N} \otimes \mathbf{Q}^{\mathrm{H}}\right)$, with $\mathbf{Q} \in \mathbb{C}^{K \times K}$ an unknown unitary matrix. In the noiseless case, this estimated channel matrix results in the ISI-free spatial mixture

$$
\mathbf{Y}=\mathbf{Q S}
$$

Analogously, the extension to the multiuser case of the subspace approach of [3] produces $\mathbf{Y}=\mathbf{A S}$, where $\mathbf{A} \in \mathbb{C}^{K \times K}$ is an arbitrary (not necessarily unitary) regular matrix [1]. Similar results are obtained in the extended direct symbol estimation method of [5], where the matrix characterizing the remaining spatial mixture is also unitary (at least for a sufficient number of symbols) [1].
In summary, the blind separation problem of instantaneous linear mixtures given by eqn. (4) needs to be solved in a second processing step. Previous works have focused on benefiting from the CM or FA properties of digital transmissions [1, 5, 7, 8, 9], but herein we opt to take advantage of the users' mutual independence. Due to the i.i.d. assumption, one must resort to ICA techniques relying on higher-order statistics (HOS). The use of HOS constrains the source components to be non-Gaussian, which is verified by most digital modulations. We select the kurtosis-based fixed-point FastICA algorithm [12] for its robustness and rapid convergence properties. Generically, assume that $C$ and $T$ denote the number of columns of $\mathbf{Q}$ and $\mathbf{Y}$, respectively. For complex-valued sources, the FastICA updating rule at iteration $k$ takes the form [11]:

1. Update: $\hat{\mathbf{Q}}_{k}=\frac{1}{T} \mathbf{Y}\left(\left|\hat{\mathbf{S}}_{k-1}\right|^{2} \odot \hat{\mathbf{S}}_{k-1}\right)^{\mathrm{H}}-2 \hat{\mathbf{Q}}_{k-1}$, with $\hat{\mathbf{S}}_{k-1}=\hat{\mathbf{Q}}_{k-1}^{\mathrm{H}} \mathbf{Y}$.

2. Symmetric decorrelation: $\hat{\mathbf{Q}}_{k} \leftarrow \hat{\mathbf{Q}}_{k}\left(\hat{\mathbf{Q}}_{k}^{\mathrm{H}} \hat{\mathbf{Q}}_{k}\right)^{-1 / 2}$.

The orthogonal projection on the set of unitary matrices of Step 2 can be efficiently carried out as $\hat{\mathbf{Q}}_{k} \leftarrow \mathbf{U V}^{\mathrm{H}}$ from the SVD $\hat{\mathbf{Q}}_{k}=\mathbf{U} \mathbf{\Sigma} \mathbf{V}^{\mathrm{H}}$. As termination criterion we choose

$$
\left|\operatorname{trace}\left(\left|\hat{\mathbf{Q}}_{k}^{\mathrm{H}} \hat{\mathbf{Q}}_{k-1}\right|\right) / C-1\right|<10^{-3} / T .
$$

In our experiments, less than $2 C$ iterations are typically required for convergence in high SNR environments. For low SNR or insufficient sample length, the algorithm may not converge, so we set the above maximum number of iterations as an additional termination test. After convergence (or termination otherwise), the channel estimate can be updated as $\hat{\mathbf{H}} \leftarrow \hat{\mathbf{H}}\left(\mathbf{I}_{M+N} \otimes \hat{\mathbf{Q}}\right)$. If a direct symbol estimation is used, the source symbols can be obtained from the final update of $\hat{\mathbf{S}}$. The channel matrix can then be estimated (if needed) via, e.g., LS fitting [5].

\section{ICA-AIDED SYMBOL DETECTION}

\subsection{MMSE-ICA Detection}

Let $\hat{\mathbf{H}}$ be the channel estimate obtained by a blind identification method. The linear MMSE detector is given by $\hat{\mathbf{s}}_{n}=\mathbf{G}^{\mathrm{H}} \mathbf{x}_{n}$, with $\mathbf{G}=\mathbf{R}_{x}^{-1} \hat{\mathbf{H}}$, where $\mathbf{R}_{x}=\mathrm{E}\left[\mathbf{x}_{n} \mathbf{x}_{n}^{\mathrm{H}}\right]$ is the sensor-output covariance matrix. The so-called subspace MMSE detector is derived from the eigenvalue decomposition of the sample estimate of $\mathbf{R}_{x}$ [10]. Let $\mathbf{W} \in \mathbb{C}^{K(M+N) \times L N}$ be the whitening matrix, i.e., a linear transformation such that $\mathbf{z}_{n}=\mathbf{W} \mathbf{x}_{n}$ fulfils $\mathbf{R}_{z}=\mathbf{I}_{K(M+N)}$. It is a simple algebraic exercise to prove that the MMSE detector adopts the equivalent alternative form $\hat{\mathbf{s}}_{n}=\tilde{\mathbf{G}}^{\mathrm{H}} \mathbf{z}_{n}$, with

$$
\tilde{\mathbf{G}}=\mathbf{W} \hat{\mathbf{H}} \text {. }
$$

The idea developed in [11] for a DS-CDMA signal model consists of carrying out the detection with the separating matrix $\tilde{\mathbf{G}}^{\prime}$ provided by an ICA algorithm initialized with $\tilde{\mathbf{G}}$. The rationale behind this notion is to refine the MMSE solution by exploiting the independence of the source components. This objective is reasonable because, as pointed out in [11], the conventional MMSE only exploits (implicitly) SOS, whereas ICA also takes into account HOS in its search for independence. Indeed, this MMSE-ICA detection scheme is shown to mitigate performance drops caused by timing, channel or model-order estimation errors in the conventional receiver [11]. The simulations of Sec. 5 demonstrate analogous features in the more general signal model of eqn. (2). 


\subsection{Simplified MMSE-ICA Detection}

The peculiarities of the FIR-MIMO model enables the simplification of the hybrid MMSE-ICA detector. In particular, the components of the vector $\mathbf{s}_{n}$ are stacked time-shifted versions of the source symbols $\mathbf{s}(n)$, and thus it suffices to estimate its first $K$ components. By virtue of eqn. (6), $\mathbf{s}(n)$ can be MMSE-detected via $\hat{\mathbf{s}}(n)=\tilde{\mathbf{G}}_{K}^{\mathrm{H}} \mathbf{z}_{n}$ where

$$
\tilde{\mathbf{G}}_{K}=\mathbf{W} \hat{\mathbf{H}}_{K}
$$

and $\hat{\mathbf{H}}_{K}$ represents the first $K$ columns of the estimated channel. Matrix $\tilde{\mathbf{G}}_{K}$ can then be used as the initial point of the ICA algorithm. Looking at the structure of the true channel matrix $\mathbf{H}$ in (3), eqn. (7) could be further simplified into $\tilde{\mathbf{G}}_{K}=\mathbf{W}_{K} \hat{\mathbf{H}}(0)$, where $\mathbf{W}_{K}$ corresponds to the first $K$ columns of the whitening matrix and $\hat{\mathbf{H}}(0)$ is an estimate of matrix $\operatorname{tap} \mathbf{H}(0)$. Although the effects of this further simplification are worth investigating, we only consider the form (7) hereafter. The relevant fact is that the number of columns to be updated is now reduced by a factor of $M+N$ with respect to matrix $\tilde{\mathbf{G}}$, with the consequent decrease in computational complexity. This simplified MMSE-ICA detection scheme is not only more computationally efficient, but also outperforms the full MMSE-ICA detector, as it will be illustrated in Sec. 5

Note that the choice of the first $K$ columns of $\hat{\mathbf{H}}$ to construct matrix $\tilde{\mathbf{G}}_{K}$ is somewhat arbitrary and may indeed be statistically suboptimal. Its advantage over other $K$-column block choices needs to be analyzed. Nevertheless, simulation results indicate that the beneficial effects of the above selection are already significant.

\subsection{Switching Strategy}

In low SNR or short sample size scenarios, the application of the ICA refinement may actually worsen the results of the conventional detector. In the CDMA environment [11], the MMSE-ICA solution was only chosen when the prior information provided by the conventional receiver was fairly preserved in the ICA refinement, i.e., when the initial and final vectors of the ICA algorithm were sufficiently correlated. We extend this criterion to the MIMO model by switching to the MMSE-ICA solution of Sec.4.1 if

$$
\operatorname{Re}\left(\operatorname{trace}\left(\tilde{\mathbf{G}}^{\mathrm{H}} \tilde{\mathbf{G}}^{\prime}\right)\right)>0.8 K(M+N)
$$

where $\mathbb{R e}(\cdot)$ denotes the real part of its complex argument, and using the conventional MMSE detector otherwise. Using a threshold of $0.8 \mathrm{~K}$, this switching rule is made applicable to the simplified MMSE-ICA solution of Sec.4.2.

\section{SIMULATION RESULTS}

We simulate a wireless communication system in which $K=5$ users transmit QPSK-modulated information across a frequencyselective channel with a maximum delay spread of $M+1=5$ symbol periods. A smoothing factor of $N=5$ combined with a spatio-temporal diversity level of $L=10$ result in a $50 \times 45$ channel matrix with a condition number of around 100 . Since, by construction, the simplified detector only estimates $\mathbf{s}(n)$ for $n=M+N-1$ to $N_{d}+M-1$, we define the signal mean square error as

$$
\mathrm{SMSE}=\frac{1}{K\left(N_{d}-N+1\right)} \sum_{n=M+N-1}^{N_{d}+M-1}\|\hat{\mathbf{s}}(n)-\mathbf{s}(n)\|^{2}
$$

although similar results are obtained with a performance criterion based on $\left\|\hat{\mathbf{s}}_{n}-\mathbf{s}_{n}\right\|^{2}$. The SMSE is averaged over $\nu$ Monte Carlo iterations, with $\nu N_{d} \geqslant 10^{5}$.

The first experiment evaluates the noise-free scenario for a varying number of observed symbol periods. For ISI cancellation, we use the extended version [4] of the blind channel identification method of [2]. This method ('TON94') is then followed by a CCI-cancellation stage based on ILSF [1] or ICA (Sec. 3). The ILSF is initialized at the identity matrix. The case of perfect channel estimation is also considered. After identifying the channel, the transmitted symbols are estimated by one of the three detection schemes explained in Sec. 4. subspace MMSE, MMSE-ICA and simplified MMSE-ICA. The latter two detectors include the switching strategy of Sec. 4.3 The results of Fig. 1 show that ICA-based CCI cancellation proves consistently superior to the FA-based approach, with up to 10-dB improvements for the simplified MMSE-ICA receiver. In addition, ICA-based CCI removal seems to effectively compensate for channel identification errors, since it reaches the performance obtained under perfect channel knowledge. Also, the ICA-assisted detectors notably improve the conventional MMSE receiver, with gains exceeding the $15 \mathrm{~dB}$ in the simplified MMSE-ICA method with ICA-based CCI suppression.

The effects of additive white complex-Gaussian noise are assessed in Fig. 2 with an average SNR per sensor component of $\operatorname{trace}\left(\mathbf{H H}^{\mathrm{H}}\right) /\left(\sigma^{2} L N\right)$ and fixed $N_{d}=500$ symbol periods. The optimal MMSE curve corresponds to the MMSE receiver with perfect channel and sensor covariance matrix estimates. Again, a superior behaviour of the ICA-based CCI-cancellation and detection techniques is evidenced.

Figs. 3 (4 consider the extended direct symbol estimation algorithm of [5] ('LIU95'), followed by the ILSF/ICA CCI-removal stage. The ICA-based approach seems to overcome the lack of consistency observed in the results of the ILSF method. Remark that the appropriate use of ICA enables channel-identification based blind equalization reach the performance of direct symbol estimation. This latter technique surpasses the optimal MMSE receiver over certain SNR range, which is an interesting outcome worthy of further investigation.

\section{CONCLUSIONS}

We have considered the exploitation of the mutual statistical independence between the users' signals for CCI cancellation and data detection in the BIE of FIR-MIMO channels. The use of ICA for CCI cancellation outperforms the FA-based ILSF algorithm, both in direct symbol estimation and channel-identification based methods. In the latter, the ICA-assisted MMSE detection strategy further increases the robustness, relative to the conventional MMSE receiver, against channel-estimation inaccuracies. The peculiar structure of the FIR-MIMO model leads to a simplified ICA-aided MMSE detector, with improved performance at a lower computational cost. As an added advantage of the ICA-based BIE methods, the prior knowledge of system parameters such as users' modulations or signature sequences is spared, with the consequent increase in versatility. These features make ICA-based techniques very promising not only in future commercial wireless systems, but also in non-cooperative military scenarios. 


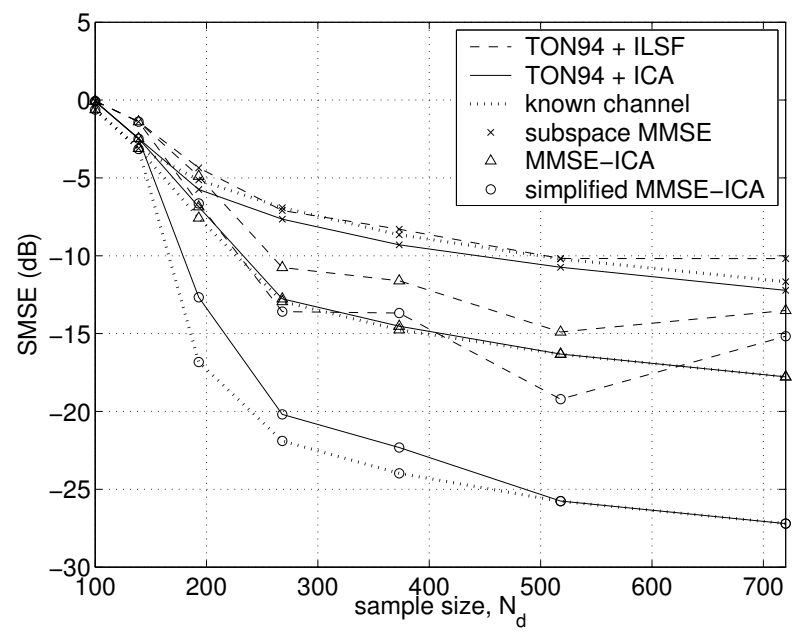

Figure 1: Detection performance vs. sample size, $\mathrm{SNR}=\infty$.

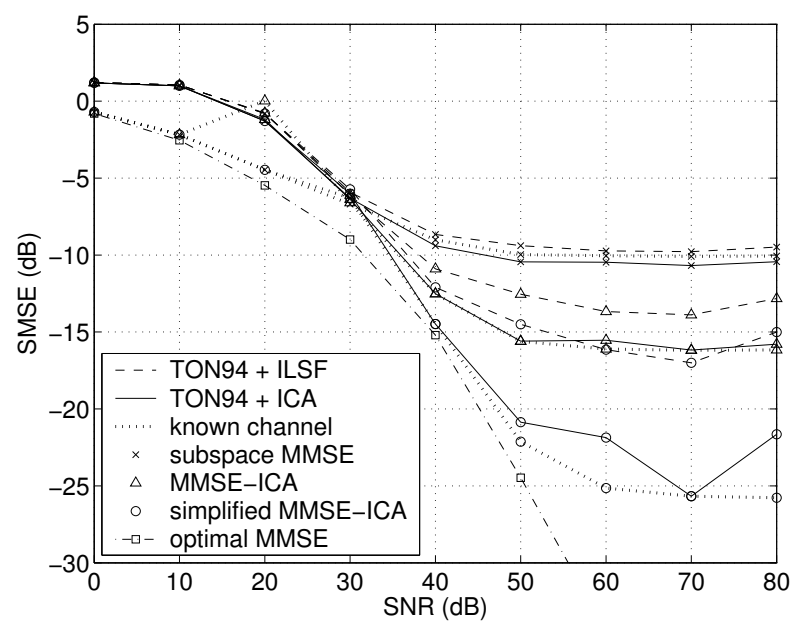

Figure 2: Detection performance vs. SNR, $N_{d}=500$.

\section{REFERENCES}

[1] A.-J. van der Veen, S. Talwar, and A. Paulraj, "A subspace approach to blind space-time signal processing for wireless communication systems," IEEE Transactions on Signal Processing, vol. 45, no. 1, pp. 173-190, Jan. 1997.

[2] L. Tong, G. Xu, and T. Kailath, "Blind identification and equalization based on second-order statistics: a time domain approach," IEEE Transactions on Information Theory, vol. 40, no. 2, pp. 340-349, Mar. 1994.

[3] E. Moulines, P. Duhamel, J.-F. Cardoso, and S. Mayrargue, "Subspace methods for the blind identification of multichannel FIR filters," IEEE Transactions on Signal Processing, vol. 43, no. 2, pp. 516-525, Feb. 1995.

[4] V. Zarzoso, A. K. Nandi, J. I. García, and L. V. Domínguez, "Blind identification and equalization of MIMO FIR channels based on second-order statistics and blind source separation," in Proc. DSP2002, 14th International Conference on Digital Signal Processing, Santorini, Greece, July 1-3, 2002, vol. I, pp. 135-138.

[5] H. Liu and G. Xu, "Closed-form blind symbol estimation in digital communications," IEEE Transactions on Signal Processing, vol. 43, no. 11, pp. 2714-2723, Nov. 1995.

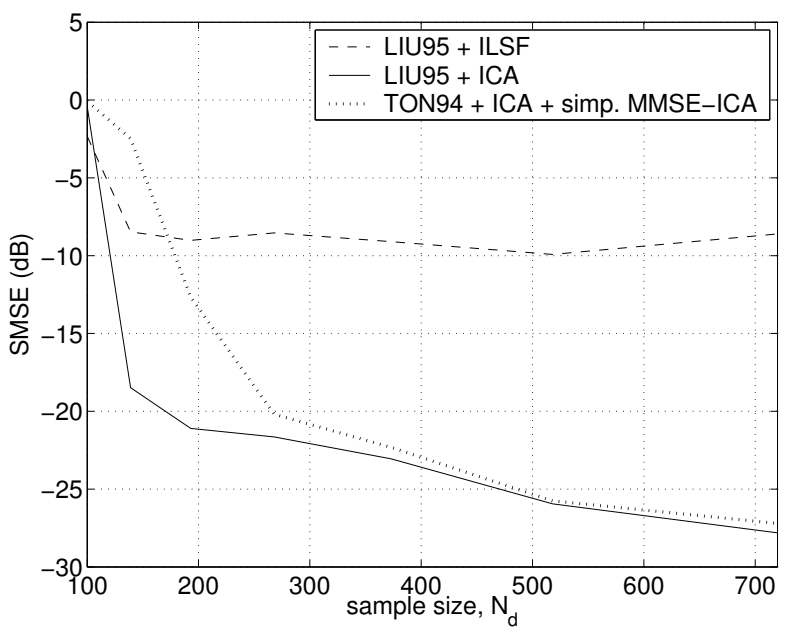

Figure 3: Detection performance vs. sample size, SNR $=\infty$.

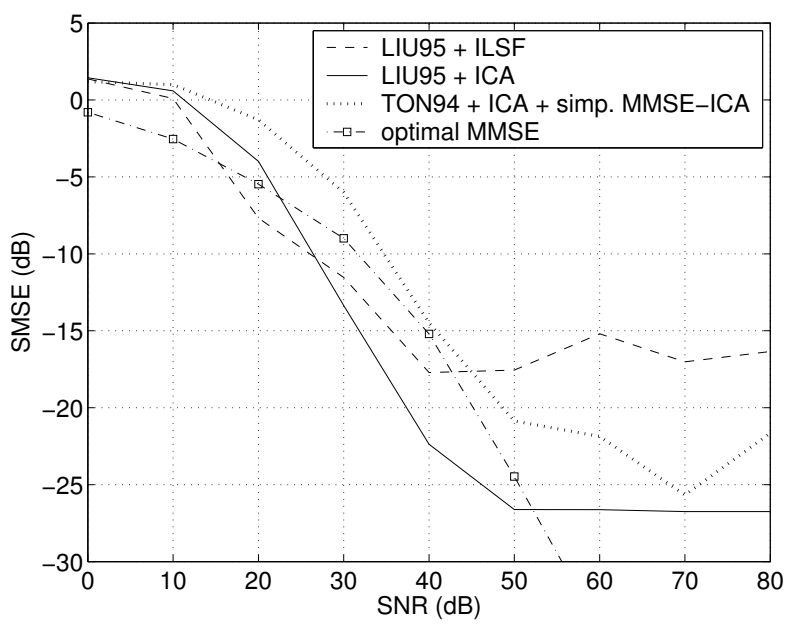

Figure 4: Detection performance vs. SNR, $N_{d}=500$.

[6] K. Abed-Meraim, P. Loubaton, and E. Moulines, "A subspace algorithm for certain blind identification problems," IEEE Transactions on Information Theory, vol. 43, no. 2, pp. 499-511, Mar. 1997.

[7] S. Talwar, M. Viberg, and A. Paulraj, "Blind separation of synchronous co-channel digital signals using an antenna array. Part I: Algorithms," IEEE Transactions on Signal Processing, vol. 44, no. 5, pp. 1184-1197, May 1996.

[8] A.-J. van der Veen and A. Paulraj, "An analytical constant modulus algorithm," IEEE Transactions on Signal Processing, vol. 44, no. 5, pp. 1136-1155, May 1996.

[9] A.-J. van der Veen, "Analytical method for blind binary signal separation," IEEE Transactions on Signal Processing, vol. 45, no. 4, pp. 1078-1082, Apr. 1997.

[10] X. Wang and H. V. Poor, "Blind equalization and multiuser detection in dispersive CDMA channels," IEEE Transactions on Communications, vol. 46, no. 1, pp. 91-103, Jan. 1998.

[11] T. Ristaniemi and J. Joutsensalo, "Advanced ICA-based receivers for block fading DS-CDMA channels," Signal Processing, vol. 82, no. 3, pp. 417-431, Mar. 2002.

[12] A. Hyvärinen and E. Oja, "A fast fixed-point algorithm for independent component analysis," Neural Computation, vol. 9, no. 7, pp. 1483-1492, 1997. 